\title{
Crystallization from Thin Films on a Hot Plate: A New Approach of Solid Surface Pattering
}

\author{
Ion Sandu1, Iuliana Urzica1, Marius Dumitru1, Ana Maria Banici1,2, Claudiu Teodor Fleaca1, \\ Florian Dumitrache ${ }^{1}$
}

${ }^{1}$ National Institute for Lasers, Plasma and Radiation Physics, Bucharest, Romania

${ }^{2}$ Faculty of Mathematics and Natural Sciences, University of Craiova, Craiova, Romania

Email: ion.sandu@inflpr.ro

How to cite this paper: Sandu, I., Urzica, I., Dumitru, M., Banici, A.M., Fleaca, C.T. and Dumitrache, F. (2019) Crystallization from Thin Films on a Hot Plate: A New Approach of Solid Surface Pattering. Journal of Materials Science and Chemical Engineering, 7, 9-18.

https://doi.org/10.4236/msce.2019.712002

Received: October 25, 2019

Accepted: December 7, 2019

Published: December 10, 2019

Copyright $\odot 2019$ by author(s) and Scientific Research Publishing Inc. This work is licensed under the Creative Commons Attribution International License (CC BY 4.0).

http://creativecommons.org/licenses/by/4.0/

\begin{abstract}
The sacrificial polymeric template usually used in lithographic methods could be replaced by crystalline materials such as ionic salts which, in some special conditions, can form arrays of single crystals. Atomic force microscopy investigations performed on such samples show their regularity and nanometric thickness. They can serve as a part of an ionic/molecular crystal grid or as mask in crystal lithography. Alternating the polarity degree of the employed solvents/dispersing media by matching materials qualities, we fabricated a sodium chromate and sulphur crystalline net, iron oxide nanoparticle grid and a sulphur single crystal array. The lack of any expensive/sophisticated technology in the production process of final devices makes this approach attractive.
\end{abstract}

\section{Keywords}

Dendrites, Interfaces, Low Dimensional Structures, Surface Processes

\section{Introduction}

The ability to produce nanostructured surfaces over macroscopic areas is likely to be of crucial importance to the integration of nanotechnology into commercial devices. The main actual techniques employed for their large scale fabrication, the light (usually ultraviolet) and electron lithography [1], start to reach their theoretical limits and even worse; they are already cost-prohibitive. About orders of magnitude cheaper than the lithographic technologies, the self-assembly [2] based approaches such as natural lithography [3] are of a growing interest in exploring their abilities. Between these two approaches seen as extreme in terms of cost, there are a multitude of other techniques [4] [5] [6] [7] [8] that try to get nanostructurted surfaces of the quality given by classical lithography and the 
cheapness of those obtained by self assembly. However, even if they look as being very different, electron and natural lithography have some common points. In electron lithography [9] a focused beam of electrons scans to draw custom shapes on a surface covered with an electron-sensitive film called a resist, followed by selectively chemical removal of irradiated or non-irradiated polymer. In self-assembly techniques, polystyrene or silica nanospheres self-assemble in 2D or 3D ordered close packed structures followed by infiltration of a desired material between nanosphere voids, its casting, followed by the wet chemical or oxidative thermal removal of nanospheres [10]. In both of these we have a sacrificial material (resist or nanosphere) which is removed after it ends its role in the surface structuring. However, if in electron or UV lithography the resist can be structured in "quasi-infinite" modes, in natural lithography the nanospheres can produce only few, square or hexagonal for $2 \mathrm{D}$ and hexagonal or cubic for $3 \mathrm{D}$ structures [11]. By using pre-patterned surfaces through electron or UV lithography, the number of ordering modes in nanosphere lithography can be increased, but unfortunately, also the final cost [12]. In the present work we propose an original aproach in which the sacrificial polymeric template could be replaced by crystaline materials such as ionic salts which, in some special conditions, can form arrays of single crystals. Known as dendritic crystals [13], they are common in polymer casting [14] or frequently observed when polymers such as gelatin [15] [16], organic molecules [17] or salts [18] are added even in small quantities to ionic solutions. Moreover, dendritic crystals of halite $\mathrm{NaCl}$, mirabilite $\mathrm{Na}_{2} \mathrm{SO}_{4} \cdot 10 \mathrm{H}_{2} \mathrm{O}$ or thenardite $\mathrm{Na}_{2} \mathrm{SO}_{4}$ were observed from saturated solution droplets without other additives, most of them growing at the outside of the droplet edge and being more or less influenced by the local humidity level [19]. Dendritic crystalline patterns can also appear by melt crystalizalization of some materials such as metallic alloys [20]. The cause which generates them is still under debate and few hypotesis were proposed. M.L. Neser observed the growing of dendritic crystals loaded at their ends with hemoglobin diluted and normal saline (0.9 wt\%) drop was evaporated. As cause she mentions "the impurities in the solution" [21]. For the mirabilite hydrated salt case reported in [19], the authors explained that "when dehydration of mirabilite occurred, the release of water of crystallization produced small, episodic pulses of water at the edge of the drop, which generated the secondary growth of efflorescence-like crystals outside the droplet". N. S. Bonn et al. report the growth of dendritic patterns at the end of $\mathrm{Na}_{2} \mathrm{SO}_{4}$ droplet drying on a hydrophilic surface due to "a mesoscopic wetting film around the contact line" [22]. G.H. Harrington et al., generate crystal patterns on smooth surfaces such as mica, glass, or calcite by using very dilute droplets of aqueous salt solution $(0.1 \mathrm{wt} \% \mathrm{NaCl})$ [23]. They consider that patterns are caused by "the liquid film ruptures" at the moment at which the drops are very thin. S. Mysura made crystallization studies from thin layers of $\mathrm{CaCl}_{2}$, $\mathrm{LiBr}$ or $\mathrm{LiCl}$ aqueous solutions and presented some conclusions about the phenomena that induced the dendrite formation [24]. Thus, he observed that when 
the liquid film height decreases, the heat transfer coefficeint can vary several times. Also, he noted a strong dependence between evaporation rate and crystal forms on crystallization rate. Moreover, he reported abnormally high crystallization rates $(30-50 \mathrm{~mm} / \mathrm{s})$ at extremely low supersaturations of solutions associated with the rapid growth of dendritic forms. Dendritic assemblies composed from inorganic nanoparticles can be obtained if the solutions of ionic salts are present. S. Paria et al. report the formation of self-assembled sulfur fractal patterns using colloidal sulfur particles (synthesized in situ by the reaction of sodium thiosulfate and different inorganic or organic acids) [25]. They concluded that "fractal patterns are influenced by the crystal morphology of salts" and, "particles alone form the 'coffee-ring' structure without forming any self-assembled fractal pattern". If the number of reports of dendrite crystals grown from ionic solutions is rather poor, molecular solutions of organic semiconductors are often used for surface patterning through solution-shearing method [26] [27] [28]. It is worth noting that very recently a mathematical scheme was reported for an anisotropic dendritic crystal growth model by coupling diffusion and heat equations [29].

The present paper shows how several types of ordered polycrystalline structures can be grown onto a solid substrate and suggests some possible applications of these. Ordered structures are grown far from equilibrium conditions where geometric confinement, temperature and concentration gradients occur. These patterned surfaces can be used in electronics as large scale integrated circuits or as masks as that in conventional optical lithography.

\section{Experimental}

\subsection{Dendrite Crystal Growth}

1) Growth in confined volumes (thin films). A thin liquid film of $4 \mu \mathrm{m}$ in thickness has been formed by vertical dipping and dragging out of a microscope glass slides in ionic salts aqueous solution (1 wt\%). The slide was horizontally kept till it dried.

2) Growth in confined volumes (thin films) on a hot plate. A thin liquid film of $4 \mu \mathrm{m}$ in thickness has been formed by vertical dipping and dragging out of a microscope glass slide in ionic salts aqueous solution (1 wt\%). The slide was horizontally deposited $(t=5-10 \mathrm{~s})$ onto a heater $\left(\mathrm{T}=300^{\circ} \mathrm{C}-500^{\circ} \mathrm{C}\right)$. A quasi-ordered array could be observed onto entire surface on optical microscope.

3) Growth on a tilted substrate. Sulfur powder was disolved in toluene near saturation. The liquid is pourred onto a tilted $\left(10^{\circ}\right)$ microscope glass slide thus it forms a thick liquid film pinned from substrate margins. After toluene natural evaporation, crystalized sulfur lines can be observed onto the slide.

\subsection{Ring Crystal Growth}

1) Growth in confined volumes (droplets) on a hot plate. Aqueus solution of $\mathrm{NaCl}(10 \mathrm{wt} \%)$ was sprayed onto a glass substrate heated at $300^{\circ} \mathrm{C}$. A large 
number of $\mathrm{NaCl}$ rings has been observed on entire surface by using optical microscope.

2) Growth in confined volumes (drops) in laser beam. A $5 \mathrm{~mm}$ in diameterdrop, of aqueus solution of $\mathrm{NaCl}(0.1 \mathrm{wt} \%)$ was deposited onto a glass substrate. A IR laser beam $(\lambda=10.6 \mu \mathrm{m}, \mathrm{P}=100 \mathrm{~W})$ pulse $(\mathrm{t}=0.2 \mathrm{~s})$ heats and explosive evaporates the $\mathrm{NaCl}$ solution drop [30]. $\mathrm{NaCl}$ ripples formed onto the glass substrate.

\subsection{Crystals as Template}

1) Crystal onto crystal array. Crystalized sulfur lines were obtained onto a glass slide by technique described in (2.1c). $\mathrm{A} \mathrm{K}_{2} \mathrm{CrO}_{4}$ was grown onto sulfur line by the technique described in (2.1a). Intersected suphur and $\mathrm{A}_{2} \mathrm{CrO}_{4}$ net was observed on optical microscope.

2) Nanoparticle array. Iron oxide nanoparticles synthesized by laser pyrolysis [31] were dispersed $\left(c_{1}=0.1 \mathrm{wt} \%\right)$ in a mixture of ethanol/toluene $\left(c_{2}=30 \%\right.$ vol.) and ultrasonicated. From this dispersion, a nanoparticle film was deposited onto a $\mathrm{K}_{2} \mathrm{CrO}_{4}$ dendritic surface obtained in (2.1.2) through dip coating technique. After film dried, it was immersed in distilled water and ultrasonicated for 5 minutes, extracted, and naturally dried again. Nanoparticle parallel lines could be seen using on optical microscope.

Structural characterization using X-ray diffraction was performed with a $\mathrm{Pa}$ nalytical X'Pert Pro MPD equipment in Brag-Bretano configuration, continuous scanning $\left(10^{\circ}-70^{\circ}\right)$ deg. $2 \theta$, with a step of $0.02^{\circ}$ and a time of $0.02^{\circ} .20 \mathrm{sec} / \mathrm{step}$ acquisition.

\section{Results and Discussions}

It is known that concentrated aqueous solutions of ionic salts form specific three dimensional single-crystals (Figure 1(a)) when water evaporates [32] [33] [34]. When crystallization takes place on a solid substrate the single-crystals appear as a collection of many isolated or partially clusterized similar crystals, only their size and numer varying. Sometime, when the solutions are highly diluted, dendritic shapes are obtained (small and flat single-crystals bonded in fractal like structures) (Figure 1(b)) [22] [35]. These dendritic self-assembled crystals are hardly reproducible (at least as shape).

It is known that temperature is an important parameter which dictates the density of nucleation and subsequent, the crystals size. By increasing the temperature during solvent evaporation such as: the glass slide is setteled on a heater (Figure 1(c)), droplets from a spray impact the glass slide setteled on a heater (Figure 1(d)), a IR laser beam pulse irradiates a drop setteled on a glass slide (Figure 1(e)), or an IR laser beam balleates a liquid film formed onto a glass slide (Figure 1(f)), we found that diluted ionic liquids films form different dendritic crystals on a large surface, every time. $\mathrm{NaCl}$ crystallizes in a beautiful fractal shape on square centimeters (Figures $1(\mathrm{a})-(\mathrm{f})$ ). Other ionic salts such as: 


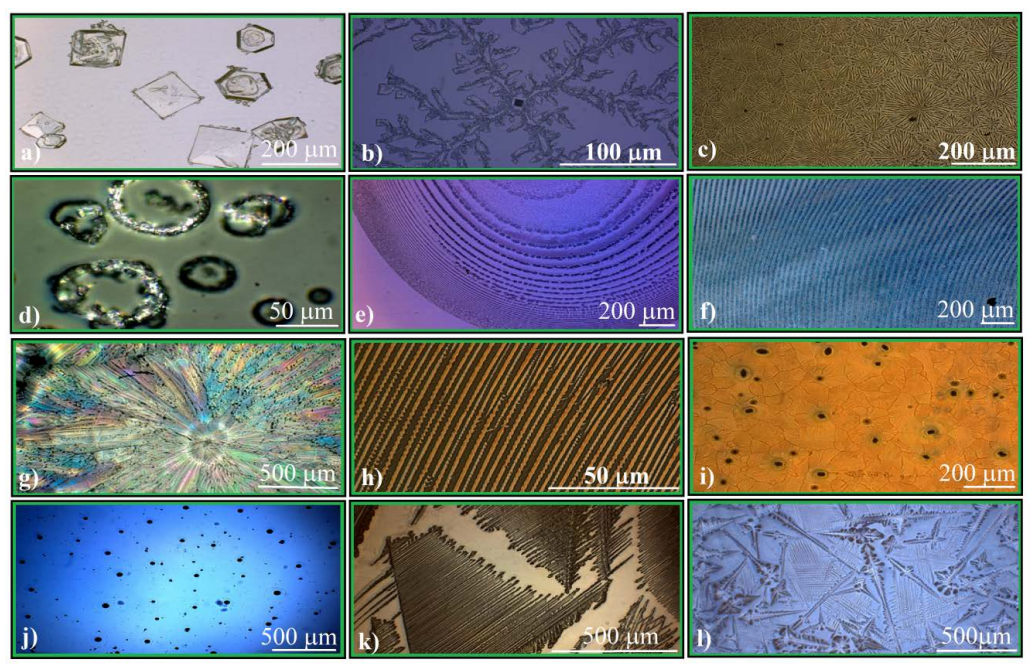

Figure 1. Optical microscopy images of aqueous $\mathrm{NaCl}$ crystallization through solvent evaporation: (a) Free evaporation of a thick film, $1 \mathrm{~mm}$ in thickness.; (b) Free evaporation of a thin film, $4 \mu \mathrm{m}$ in thickness; (c) Rapid evaporation of a thin film on a hot plate; (d) Spray droplets impacting a hot substrate; (e) IR laser beam pulse ablation of a drop; (f) Pulsed IR laser beam ablation of a thin film. Optical microscopy images of different materials crystallization from thin film solution on a hot plate: (g) sugar; (h) $\mathrm{K}_{2} \mathrm{CrO}_{4}$; (i) Tris(acetylacetonato)iron(III); (j) Ferocene; (k) Mixture of $\mathrm{NaCl}$ and sucrose.

$\mathrm{BaCl}_{2}, \mathrm{CuCl}_{2}, \mathrm{Na}_{2} \mathrm{SiO}_{3}, \mathrm{Ni}\left(\mathrm{CH}_{3} \mathrm{COO}\right)_{2}$, and others give rise to specific crystal fractals. Different ionic salts form different shapes of dendritic crystals (Figures $1(\mathrm{~g})$-(i)), the most interesting we found to be the grid which forms through $\mathrm{K}_{2} \mathrm{CrO}_{4}$ crystallization (Figure $1(\mathrm{~h})$ ). We found that these parallel and long (few $\mathrm{mm}$ ) of micrometers wide crystals could be used as template or as mask in a lithography process. Molecular solutions such as 9,10 -bis (phenylethynyl) anthracene (BPEA) used by Y. Li et al. in [8] can be able to form single crystal grid through solution-shearing by using a heater. Our atempt in repeating their experiments, by using sulfur dissolved in toluene, failed because sulphur crystallizes as micrometric spheroidal crystals (orthorhombic phase containing $\mathrm{S}_{8}$ molecules ) when solution fast evaporates (Figure $1(j)$ ). However, by keeping their idea of tilting the substrate but using a free evaporation, a concentrated solution, and a tick liquid film $(2 \mathrm{~mm})$ we succeed to obtain long sulfur single crystals in a parallel array (Figure 1(k) and Figure 2(a), top image).

By using as template sulfur or $\mathrm{K}_{2} \mathrm{CrO}_{4}$ grids we may pattern surfaces in a useful way thus some devices to be fabricated. We shall forward present three of these. If onto the surface from Figure 2(a) we form a thin $\mathrm{K}_{2} \mathrm{CrO}_{4}$ solution film and evaporate it on a hot plate, we will not obtain a $\mathrm{K}_{2} \mathrm{CrO}_{4}$ polycrystalline gride as in Figure 2(a) (middle image), but a superposed sulfur and $\mathrm{K}_{2} \mathrm{CrO}_{4}$ net as in Figure 2(a) (bottom image), a net able to diffract the white light. Keeping the approach but changing the basic materials we could form such net from much more interesting materials. In a second example, onto a $\mathrm{K}_{2} \mathrm{CrO}_{4}$ grid formed on a hot plate (Figure 2(b), top image), is deposited a thin film of iron oxide nanoparticles (Figure 2(b), middle image). After sinking in water, the $\mathrm{K}_{2} \mathrm{CrO}_{4}$ grid 

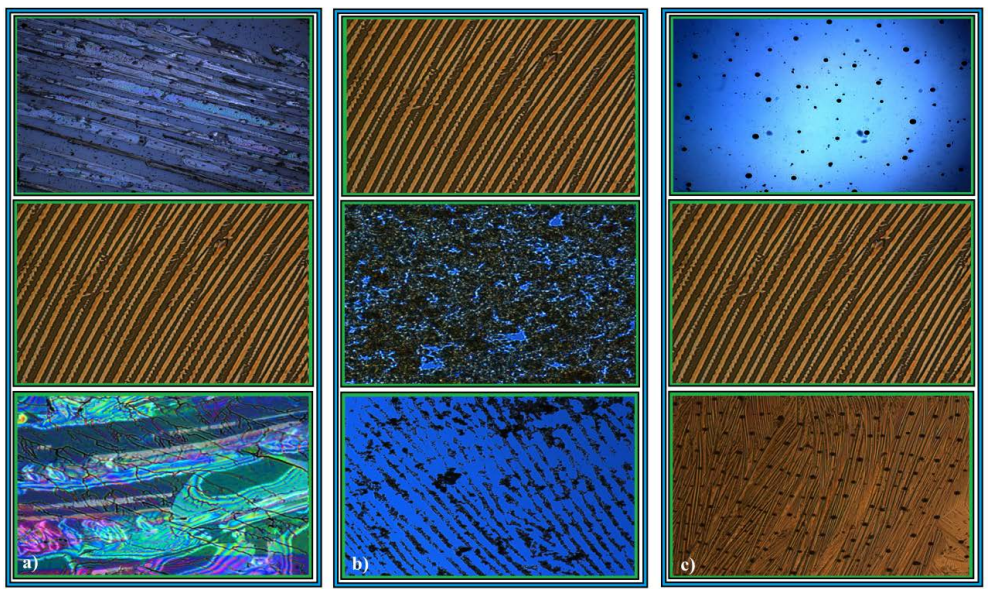

Figure 2. Optical microscopy images of: (a) Sulphur grid, top image, $\mathrm{K}_{2} \mathrm{CrO}_{4}$, grid, middle image, sulphur and $\mathrm{K}_{2} \mathrm{CrO}_{4}$, intersected net, bottom image, (b) $\mathrm{K}_{2} \mathrm{CrO}_{4}$, grid, top image, iron oxide nanoparticle film onto $\mathrm{K}_{2} \mathrm{CrO}_{4}$, grid, middle image, iron oxide nanoparticle grid, bottom image, (c) Sulphur dots on glass slide, top image, $\mathrm{K}_{2} \mathrm{CrO}_{4}$, grid, middle image, sulphur dots array self-assembeled onto a $\mathrm{K}_{2} \mathrm{CrO}_{4}$, grid, bottom image.

together with all the nanoparticles deposited on it is removed, remaining thus only the nanoparticles (as a grid) which were in close contact with the glass substrate (Figure 2(b), bottom image). In the third example, we show how a dilluted sulfur solution deposited onto a $\mathrm{K}_{2} \mathrm{CrO}_{4}$ grid (Figure 2(c), middle image) forms a sulphur dots array (Figure 2(c), bottom image) which otherway would randomly deposit onto a clean glass surface (Figure 2(c), top image).

Even if are less impresing as accuracy, these three examples can be improved and extrapolated to some interesting devices or to the study of complex phenomena in which surfaces, interfaces, and far from equilibrium crystallization lead to unexpected observations.

A such information is revealed by $\mathrm{X}$ ray diffraction (Figure 3) performed on the structures sown in Figure 2(a). If $\mathrm{K}_{2} \mathrm{CrO}_{4}$ and sulphur grids are separately grown on a flat substrate, both of them form textured surfaces in which their crystallographic orientations have some preferred directions. If the surface is not flat but structured itself $\left(\mathrm{K}_{2} \mathrm{CrO}_{4}\right.$ grid for example), the material which will crystalyze on such surface (sulphur in our case) will gain new crystallographic orientations. We consider that it would be useful to control the crystallographic orientation of a cristalline growth film trough the structure and morphology of the substrate.

As time as dendritic structures can be grown at normal temperatures (before boiling temperature) the role of high temperatures (above boiling point), seems to resume to the creation of a large number of non-equilibrium space and time zones, in which the substrate dewetting is somehow correlate with the ionic salt concentration of crystallization. The existence of some important temperature dependent parameters such as: liquid film thickness, ionic salts concentration of saturation, or evaporation rate, which act simultaneously, make difficult the finding of such correlations. However, we observe that not the absolute value of 


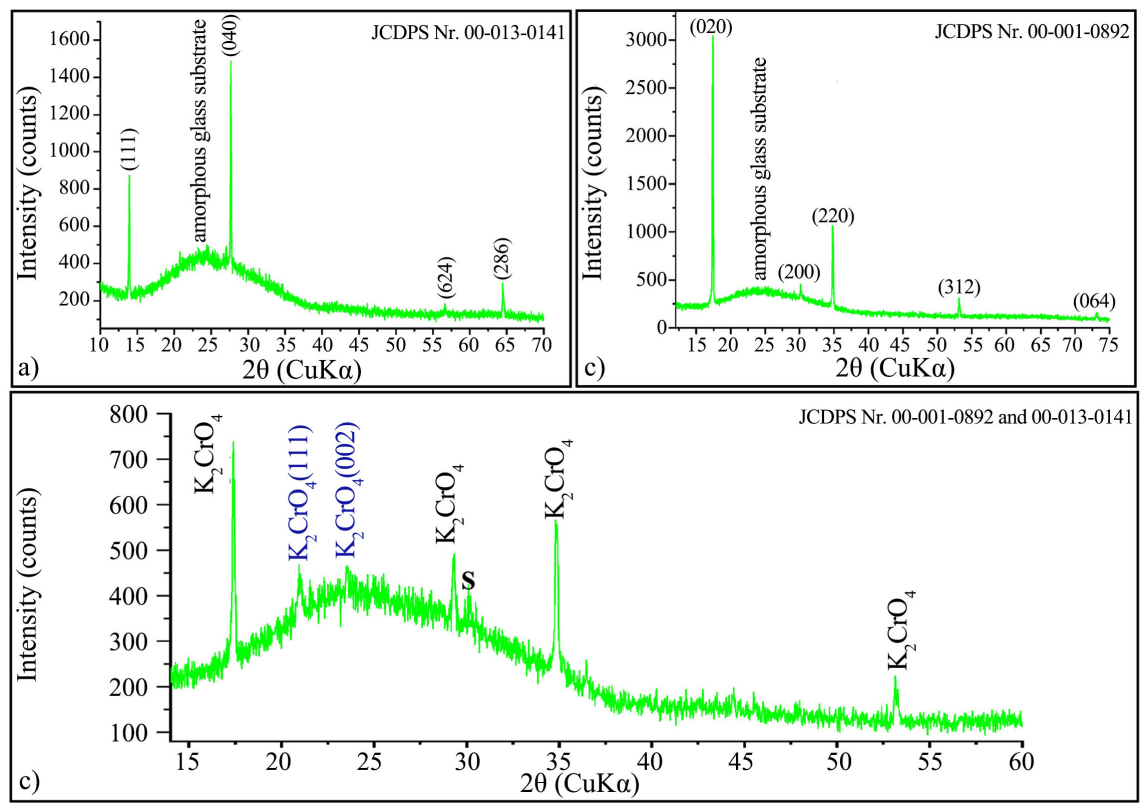

Figure 3. X ray diffractograms: (a) Sulphur grid, (b) $\mathrm{K}_{2} \mathrm{CrO}_{4}$, grid, (c) Sulphur and $\mathrm{K}_{2} \mathrm{CrO}_{4}$, intersected net.

a high temperature is important but rather the intensity (spatial and temporal) of the temperature gradient. This temperature gradient may correlate also with the texturation phenomenon found by optical and XRD measurements, thus it must be taken in account in experiments regarding surface patterning, complex systems or, non-equilibrium processes.

\section{Conclusion}

Diluted ionic salt solutions as thin films on a hot plate, crystallize in dendritic or ordered patterns which can be used as template for the crystallization or deposition of other materials. Alternating the polarity of the liquid dissolving/dispersing medium by matching materials qualities, sodium chromate and sulphur cristalline net, iron oxide nanoparticle grid and sulphur single crystal array can be fabricated.

\section{Acknowledgements}

This work was developed with the support of Ministry of Research and Innovation MCI, and carried out through the Nucleu program project no. 3N/2019, and project 46PCCDI/2018.

\section{Conflicts of Interest}

The authors declare no conflicts of interest regarding the publication of this paper.

\section{References}

[1] Hansen, M.G., Thamdrup, L.H., Mironov, A. and Kristensen, A. (2007) Combined 
Electron Beam and UV Lithography in SU-8. Microelectronic Engineering, 84, 1058-1061. https://doi.org/10.1016/j.mee.2007.01.084

[2] Whitesides, G.M. and Grzybowski, B. (2002) Self-Assembly at All Scales. Science, 2095, 2418-2421. https://doi.org/10.1126/science.1070821

[3] Haynes, C.L. and Van Duyne, R.P. (2001) Nanosphere Lithography: A Versatile Nanofabrication Tool for Studies of Size-Dependent Nanoparticle Optics. The Journal of Physical Chemistry B, 105, 5599-5611. https://doi.org/10.1021/jp010657m

[4] Tang, C., Tracz, A., Kruk, M., Zhang, R., Smilgies, D.-M., Matyjaszewski, K. and Kowalewski, T. (2005) Long-Range Ordered Thin Films of Block Copolymers Prepared by Zone-Casting and Their Thermal Conversion into Ordered Nanostructured Carbon. Journal of the American Chemical Society, 127, 6918-6919. https://doi.org/10.1021/ja0508929

[5] Santhanam, V. and Andres, R.P. (2004) Microcontact Printing of Uniform Nanoparticle Arrays. Nano Letters, 4, 41-44. https://doi.org/10.1021/nl034851r

[6] Breton, M. (1981) Formation and Possible Applications of Polymeric Langmuir-Blodgett Films. A Revifew. Journal of Macromolecular Science, Part C, 21, 61-87. https://doi.org/10.1080/00222358108080925

[7] Darhuber, A.A., Troian, S.M., Davis, J.M. and Miller, S.M. (2000) Selective Dip-Coating of Chemically Micropatterned Surfaces. Journal of Applied Physics, 88, 5119-5126. https://doi.org/10.1063/1.1317238

[8] Li, Y., Ji, D., Liu, J., Yiao, Y., Fu, X., Zhu, W., Xu, C., Dong, H., Li, J. and Hu, W. (2014) Quick Fabrication of Large-Area Organic Semiconductor Single Crystal Arrays with a Rapid Annealing Self-Solution-Shearing Method. Scientific Reports, 5, Article No. 13195. https://doi.org/10.1038/srep13195

[9] Thompson, L.F. and Kerwin, R.E. (1976) Polymer Resist Systems for Photo- and Electron Lithography. Annual Review of Material Science, 6, 267-301.

https://doi.org/10.1146/annurev.ms.06.080176.001411

[10] Armstrong, E. and O’Dwye, C. (2015) Artificial Opal Photonic Crystals and Inverse Opal Structures-Fundamentals and Applications from Optics to Energy Storage. Journal of Materials Chemistry C, 3, 6109-6143. https://doi.org/10.1039/C5TC01083G

[11] Rybin, M.V., Samusev, K.B., Lukashenko, S.Y., Kivshar, Y.S. and Limonov, M.F. (2016) Transition from Two-Dimensional Photonic Crystals to Dielectric Metasurfaces in the Optical Diffraction with a Fine Structure. Scientific Reports, 6, Article No. 30773. https://doi.org/10.1038/srep30773

[12] Wang, X., Xu, S., Cong, M., Li, H., Gu, Y. and Xu, W. (2012) Hierarchical Structural Nanopore Arrays Fabricated by Pre-Patterning Aluminum Using Nanosphere Lithography. Small, 8, 972-976. https://doi.org/10.1002/smll.201102274

[13] Glicksman, M.E. and Lupulescu, A.O. (2004) Dendritic Crystal Growth in Pure Materials. Journal of Crystal Growth, 264, 541-549. https://doi.org/10.1016/j.jcrysgro.2003.12.034

[14] Geil, P.H. and Reneker, D.H. (1961) Morphology of Dendritic Polyethylene Crystals. Journal of Polymer Science, 51, 569-582. https://doi.org/10.1002/pol.1961.1205115613

[15] Duttaa, T., Giri, A., Choudhury, M.D. and Tarafdar, S. (2013) Experiment and Simulation of Multifractal Growth of Crystalline $\mathrm{NaCl}$ Aggregates in Aqueous Gelatin Medium. Colloids and Surfaces A, 432, 127-131. https://doi.org/10.1016/j.colsurfa.2013.04.040 
[16] Goto, M., Oaki, Y. and Imai, H. (2016) Dendritic Growth of NaCl Crystals in a Gel Matrix: Variation of Branching and Control of Bending. Crystal Growth \& Design, 16, 4278-4284. https://doi.org/10.1021/acs.cgd.6b00323

[17] Ma, Y., Qi, L., Ma, J. and Cheng, H. (2004) Hierarchical, Star-Shaped PbS Crystals Formed by a Simple Solution Route. Crystal Growth \& Design, 4, 351-354. https://doi.org/10.1021/cg034174e

[18] Agrawal, V.V., Kulkarni, G.U. and Rao, C.N.R. (2008) Surfactant-Promoted Formation of Fractal and Dendritic Nanostructures of Gold and Silver at the Organic-Aqueous Interface. Journal of Colloid and Interface Science, 318, 501-506. https://doi.org/10.1016/j.jcis.2007.10.013

[19] Rodriguez-Navarro, C. and Doehne, E. (1999) Salt Weathering: Influence of Evaporation Rate, Supersaturation and Crystallization Pattern. Earth Surface Processes and Landforms, 24, 191-209. https://doi.org/10.1002/(SICI)1096-9837(199903)24:3<191::AID-ESP942>3.0.CO;2-G

[20] Haxhimali, T., Karma, A., Gonzales, F. and Rappaz, M. (2006) Orientation Selection in Dendritic Evolution. Nature Materials, 5, 660-664. https://doi.org/10.1038/nmat1693

[21] Neser, M.L. (1969) Brief Report: A Note on Hypertonic Saline Hemolysis of Red Cells. Blood, 33, 479-484. https://doi.org/10.1182/blood.V33.3.479.479

[22] Bonn, N.S., Rafa, S., Bonn, D. and Wegdam, G. (2008) Salt Crystallization during Evaporation: Impact of Interfacial Properties. Langmuir, 24, 8599-8605. https://doi.org/10.1021/la8005629

[23] Harrington, G.F., Campbell, J.M. and Christenson, H.K. (2013) Crystal Patterns Created by Rupture of a Thin Film. Crystal Growth \& Design, 13, 5062-5067. https://doi.org/10.1021/cg401235f

[24] Misyura, S.Y. (2018) Evaporation and Heat Transfer of Aqueous Salt Solutions during Crystallization. Applied Thermal Engineering, 139, 203-212. https://doi.org/10.1016/j.applthermaleng.2018.04.068

[25] Paria, S., Chaudhuri, R.G. and Jason, N.N. (2014) Self-Assembly of Colloidal Sulfur Particles on a Glass Surface from Evaporating Sessile Drops: Influence of Different Salts. New Journal of Chemistry, 38, 5943-5951. https://doi.org/10.1039/C4NJ01267D

[26] Aizenberg, J. (2004) Cristalization in Patterns: A Bio-Inspired Approach. Advanced Materials, 16, 1295-1302. https://doi.org/10.1002/adma.200400759

[27] Giri, G., Miller, E. and Baoa, Z. (2014) High-Resolution X-Ray Analysis of Graphene Grown on 4H-SiC (0001) at Low Pressures. Journal of Materials Research, 29, 439-446. https://doi.org/10.1557/jmr.2014.305

[28] Diao, Y., Tee, B.C.-K., Giri, G., Xu, J., Kim, D.H., Becerril, H.A., Stoltenberg, R.M., Lee, T.H., Xue, G., Mannsfeld, S.C.M. and Bao, Z. (2013) Solution Coating of Large-Area Organic Semiconductor Thin Films with Aligned Single-Crystalline Domains. Nature Materials, 12, 665-671. https://doi.org/10.1038/nmat3650

[29] Zhang, J., Chen, C. and Yang, X. (2019) A Novel Decoupled and Stable Scheme for an Anisotropic Phase-Field Dendritic Crystal Growth Model. Applied Mathematics Letters, 95, 122-129. https://doi.org/10.1016/j.aml.2019.03.029

[30] Sandu, I., Urzica, I., Niculescua, A.M., Fleaca, C.T., Dumitrache, F. and Badiceanu, M. (2017) Self-Organisation of Single-Crystals as Ripple Patterns through Laserablation of Ionic Salt Solutions. Applied Surface Science, 417, 160-164. https://doi.org/10.1016/j.apsusc.2017.02.104

[31] Dumitrache, F., Morjan, I., Fleaca, C., Badoi, A., Manda, G., Pop, S., Marta, D.S., 
Huminic, G., Huminic, A., Vekas, L., Daia, C., Marinica, O., Luculescu, C. and Niculescu, A.-M. (2015) Highly Magnetic $\mathrm{Fe}_{2} \mathrm{O}_{3}$ Nanoparticles Synthesized by Laser Pyrolysis Used for Biological and Heat Transfer Applications. Applied Surface Science, 336, 297-303. https://doi.org/10.1016/j.apsusc.2014.12.098

[32] Garcia-Ruiz, J.M. (2003) Nucleation of Protein Crystals. Journal of Structural Biology, 142, 22-31. https://doi.org/10.1016/S1047-8477(03)00035-2

[33] Merikanto, J., Zapadinsky, E., Lauri, A. and Vehkamaki, H. (2007) Origin of the Failure of Classical Nucleation Theory: Incorrect Description of the Smallest Clusters. Physical Review Letters, 98, 1-4. https://doi.org/10.1103/PhysRevLett.98.145702

[34] Volmer, M. (1939) Kinetik der Phasenbildung. Steinkopff, Dreesden und Leipzig.

[35] Wu, H. and Briscoe, W.H. (2018) Morphogenesis of Polycrystalline Dendritic Patterns from Evaporation of a Reactive Nanofluid Sessile Drop. Physical Review Materials, 2, Article ID: 04560. https://doi.org/10.1103/PhysRevMaterials.2.045601 